\title{
Solution stationnaire de l'équation de coagulation de gouttelettes en chute avec le vent horizontal
}

\author{
Meriem Merad (*) - Hanane Belhireche (*) - Hisao Fujita Yashima (**)
}

ABstRaCT - We consider the equation which describes the coagulation process of water drops which fall in the air. The equation is considered in a two-dimensional domain and the density of water drops entering the domain is supposed to be given. Under the hypothesis of the constancy of the horizontal wind and some suitable conditions, we prove the existence and the uniqueness of the stationary solution.

RÉSUMÉ - On considère l'équation décrivant le processus de coagulation des gouttelettes qui tombent dans l'air. L'équation est considérée dans un domaine de dimension 2 et la densité des gouttelettes à l'entrée du domaine est supposée donnée. Sous l'hypothèse que la vitesse de l'air est constante dans la direction horizontale et quelques autres conditions convenables, on prouve l'existence et l'unicité de la solution stationnaire.

Mathematics Subject Classification (2010). 45K05, 45G10, 86A10.

KEYworDS. Integro-differential equations, coagulation of droplets, fall of droplets.

\section{Introduction}

Comme il est bien connu, des nuages formés dans l'atmosphère, les gouttelettes suffisamment grandes vont tomber comme pluie, ce qui démontre le rôle essentiel du processus de coagulation des gouttelettes dans

(*) Indirizzo degli A.: Laboratoire de Mathématiques appliquées et de modélisation, Université 8 mai 1945, Guelma, Algérie.

E-mail:mrad.meriem@gmail.com hanane.belhireche@gmail.com

(**) Indirizzo dell'A.: Laboratoire de Mathématiques appliquées et de modélisation, Université 8 mai 1945, Guelma, Algérie et Dipartimento di Matematica, Università di Torino, Torino, Italia.

E-mail: hisao.fujitayashima@unito.it 
l'apparition de la pluie. L'équation dite équation de Smoluchowski, proposée par Smoluchowski (voir [16]) et Müller (voir [11]), décrit le processus de coagulation; cependant cette équation dans sa version communément considérée ne tient pas compte de l'effet de la chute des gouttelettes. Malgré que l'équation de Smoluchowski ait été étudiée par plusieurs auteurs (voir [17], [9], [3], [10], [5], [4], [12], etc...), à notre connaissance la description mathématique du processus de coagulation des gouttelettes dans leur déplacement et en particulier le déplacement dû à la force gravitationnelle n'est pas encore bien élucidée.

Dans ce présent travail nous allons considérer des gouttelettes qui, se coagulant avec une certaine probabilité, tombent avec une vitesse qui sera déterminée par la force gravitationnelle, la friction entre ces gouttelettes et l'air ainsi que la vitesse de ce dernier. Les gouttelettes considérées doivent être distribuées selon la masse $m$ de chacune d'elles, tandis que la friction avec l'air, ainsi que la probabilité de coagulation, dépend de la masse $m$. Ici nous nous limitons à considérer l'état stationnaire avec un vent horizontal constant, en renvoyant l'analyse de cas plus généraux aux études futures.

Du point de vue technique, nous allons considérer une équation intégrodifférentielle pour une fonction inconnue $\sigma=\sigma(m, x, z)$ représentant la densité (par rapport au volume de l'air) de l'eau liquide contenue dans les gouttelettes de masse $m$. Nous considérons $\sigma$ comme une fonction dépendante de la masse (de la gouttelette) $m$ et de la position $(x, z) \in \mathbb{R}^{2}$; comme nous supposons que le mouvement de l'air en considération est un vent horizontal constant, la position dans la direction horizontale et orthogonale à la direction du vent ne nous intéresse pas particulièrement. L'équation dans la forme précise va être formulée dans le paragraphe suivant (voir (2.4)).

Dans ce qui suit, nous allons démontrer l'existence et l'unicité de la solution de l'équation avec une condition aux limites dans les cas respectivement de l'absence du vent (vitesse du vent nulle) et de la présence du vent (vitesse du vent non nulle). Nous espérons que l'ultérieure analyse donnera des caractérisations plus intéressantes du point de vue physique.

\section{Position du problème}

Désignons par $\sigma(m, x, z, t)$ la densité de l'eau liquide contenue dans les gouttelettes de masse $m$ au point $(x, z) \in \Omega\left(\subset \mathbb{R}^{2}\right)$ à l'instant $t \in \mathbb{R}$, c'està-dire, la masse de l'eau liquide contenue dans les gouttelettes de masse $m$ 
qui se trouvent dans l'unité de volume de l'air. Le nombre, au sens purement statistique, des gouttelettes de masse $m$ dans l'unité de volume sera alors donné par

$$
\tilde{n}(m, x, z, t)=\frac{\sigma(m, x, z, t)}{m} .
$$

L'équation de Smoluchowski est normalement formulée par rapport au nombre $\tilde{n}=\tilde{n}(m, t)$ de gouttelettes de masse $m$. Mais nous préférons utiliser la densité $\sigma$ pour la commodité pour la modélisation générale des phénomènes météorologiques (voir [6], [1], [14]).

Nous allons considérer la densité $\sigma$ dans le domaine

$$
\Omega=\mathbb{R} \times] 0,1\left[=\left\{(x, z) \in \mathbb{R}^{2} \mid 0<z<1\right\} .\right.
$$

On va considérer également la vitesse des gouttelettes qui se déplacent à cause de la force gravitationnelle et du mouvement de l'air dans lequel elles se trouvent. Comme l'effet de la friction entre les gouttelettes et l'air dépend sensiblement de la masse de chaque gouttelette, la vitesse des gouttelettes doit être en fonction de la masse $m$. Nous admettons que la vitesse $u=u(m)$ d'une gouttelette de masse $m$ est donnée par

$$
u=u(m)=\left(\bar{v},-\frac{g}{\alpha(m)}\right),
$$

où $\bar{v}$ et $g$ sont des constantes $(\bar{v} \in \mathbb{R}, g>0)$, tandis que $\alpha(m)$ est une fonction de la masse $m(\alpha(m)>0)$. Si $g, \alpha(m)$ et $\bar{v}$ désignent respectivement l'accélération gravitationnelle, le coefficient de friction entre les gouttelettes et l'air et la vitesse de l'air (dans la direction de l'axe $x$ ), la relation (2.2) correspond, dans une bonne approximation, à la vitesse réelle des gouttelettes dans l'atmosphère (voir par exemple [15], [1], [14]).

Si nous considérons la variation de $\sigma(m, x, z, t)$ due au déplacement avec la vitesse $u(m)$ des gouttelettes et au processus de coagulation, nous aurons

$$
\begin{aligned}
\partial_{t} \sigma(m, x, z, t) & +\nabla_{(x, z)} \cdot(\sigma(m, x, z, t) u(m))= \\
= & \frac{m}{2} \int_{0}^{m} \beta\left(m-m^{\prime}, m^{\prime}\right) \sigma\left(m^{\prime}, x, z, t\right) \sigma\left(m-m^{\prime}, x, z, t\right) d m^{\prime}+ \\
& -m \int_{0}^{\infty} \beta\left(m, m^{\prime}\right) \sigma(m, x, z, t) \sigma\left(m^{\prime}, x, z, t\right) d m^{\prime},
\end{aligned}
$$


où $\nabla_{(x, z)}=\left(\partial_{x}, \partial_{z}\right)$, tandis que $\beta\left(m_{1}, m_{2}\right)$ représente la probabilité de rencontre entre une gouttelette de masse $m_{1}$ et une gouttelette de masse $m_{2}$ (avec la valeur de la probabilité normalisée par rapport à la masse). Si dans l'équation (2.3) on néglige la dépendance de $(x, z) \in \Omega$, l'équation sera réduite à l'équation de Smoluchowski (dans une version avec la densité $\sigma(m, t)=m \tilde{n}(m, t))$.

En renvoyant l'étude de l'équation d'évolution (2.3) à des recherches futures, dans le présent travail nous allons nous occuper du cas stationnaire, c'est-à-dire, nous allons considérer l'équation

$$
\begin{aligned}
& \nabla_{(x, z)} \cdot(\sigma(m, x, z) u(m))= \\
& =\frac{m}{2} \int_{0}^{m} \beta\left(m-m^{\prime}, m^{\prime}\right) \sigma\left(m^{\prime}, x, z\right) \sigma\left(m-m^{\prime}, x, z\right) d m^{\prime}+ \\
& \quad-m \int_{0}^{\infty} \beta\left(m, m^{\prime}\right) \sigma(m, x, z) \sigma\left(m^{\prime}, x, z\right) d m^{\prime}
\end{aligned}
$$

avec la condition

$$
\sigma(m, x, 1)=\bar{\sigma}(m, x) .
$$

Comme les gouttelettes tombent de $\{z=1\}$ vers $\{z=0\}$ avec la vitesse $u=u(m)$ (voir (2.2)), la condition (2.5) est une condition "initiale" (ou condition d'entrée) pour les gouttelettes qui partent de la position $(x, 1)$.

On rappelle que dans la Nature, à cause de la courbure très élevée de la surface, les gouttelettes très petites s'évaporent immédiatement (voir par exemple [13], [7]) et que d'autre part les gouttelettes très grandes se fragmentent à cause de la friction avec l'air environnant. Pour cela, nous nous intéressons à la fonction de densité $\sigma(m, x, z)$ avec $m$ entre deux extrémités $\bar{m}_{a}$ et $\bar{m}_{A}$,

$$
0<\bar{m}_{a} \leq m \leq \bar{m}_{A}<\infty .
$$

En ce qui concerne la fonction $\alpha(m)$, qui représenterait l'effet de la friction entre les gouttelettes et l'air, dans le présent travail nous supposons que $\alpha(m)$ est une fonction strictement positive et suffisamment régulière (par exemple $\alpha(m) \in C^{1}\left(\mathbb{R}_{+}\right)$). Il est utile de rappeler que dans l'état normal de l'atmosphère $\alpha(m)$ est une fonction décroissante et ses valeurs varient sensiblement selon les valeurs de $m$ (pour les données exprimentales, voir par exemple [15]). Même si l'effet de la friction (par l'unité de masse) croît rapidement quand $m$ s'approche de 0 , compte tenu de l'absence de gouttelettes très petites $\left(m<\bar{m}_{a}\right)$, pour éviter le 
raisonnement inutilement compliqué, nous supposons que

$$
\sup _{m \in \mathbb{R}_{+}} \alpha(m)<\infty \text {. }
$$

Pour la fonction $\beta\left(m_{1}, m_{2}\right)$ nous supposons que

$$
\begin{gathered}
\beta(\cdot, \cdot) \in C\left(\mathbb{R}_{+} \times \mathbb{R}_{+}\right), \quad \beta\left(m_{1}, m_{2}\right) \geq 0 \quad \forall\left(m_{1}, m_{2}\right) \in \mathbb{R}_{+} \times \mathbb{R}_{+}, \\
\beta\left(m_{1}, m_{2}\right)=\beta\left(m_{2}, m_{1}\right) \\
\beta\left(m_{1}, m_{2}\right)=0 \quad \text { pour } m_{1}+m_{2} \geq \bar{m}_{A} .
\end{gathered}
$$

Les conditions (2.6) et (2.7) sont des conditions naturelles de la fonction de probabilité de rencontre de gouttelettes. D'autre part, la condition (2.8) est une approximation motivée par le fait que, comme nous l'avons déjà évoqué, dans l'atmosphère les grandes gouttelettes subissent également le processus de fragmentation, qui contrebalance la croissance de la population de gouttelettes de masse élevée due à la coagulation (cette approximation a été adoptée même dans [6], [1], [14]).

\section{Cas de l'absence du mouvement de l'air}

Dans le cas où $\bar{v}=0$, le problème (2.4)-(2.5) se réduit à une famille de problèmes dans le domaine $0<z<1$, paramétrisée par $x \in \mathbb{R}$. En effet, si $\bar{v}=0, u(m)$ se réduit à

$$
u(m)=\left(0,-\frac{g}{\alpha(m)}\right),
$$

ce qui nous permet d'envisager le problème (2.4)-(2.5) séparément pour chaque $x \in \mathbb{R}$. Donc, en posant $\bar{\sigma}(m)=\bar{\sigma}(m, x)$ pour chaque $x \in \mathbb{R}$ et en écrivant $\sigma(m, z)$ au lieu de $\sigma(m, x, z)$, nous avons à considérer

$$
\begin{gathered}
-\partial_{z}\left(\sigma(m, z) \frac{g}{\alpha(m)}\right)=\frac{m}{2} \int_{0}^{m} \beta\left(m-m^{\prime}, m^{\prime}\right) \sigma\left(m^{\prime}, z\right) \sigma\left(m-m^{\prime}, z\right) d m^{\prime}+ \\
\quad-m \int_{0}^{\infty} \beta\left(m, m^{\prime}\right) \sigma(m, z) \sigma\left(m^{\prime}, z\right) d m^{\prime}, \\
\sigma(m, 1)=\bar{\sigma}(m) .
\end{gathered}
$$

Comme $\alpha(m)$ ne dépend pas de $z$, l'équation (3.1) peut être écrite dans 
la forme

$$
\begin{array}{r}
\partial_{z} \sigma(m, z)=-\frac{m \alpha(m)}{2 g} \int_{0}^{m} \beta\left(m-m^{\prime}, m^{\prime}\right) \sigma\left(m^{\prime}, z\right) \sigma\left(m-m^{\prime}, z\right) d m^{\prime}+ \\
+\frac{m \alpha(m)}{g} \int_{0}^{\infty} \beta\left(m, m^{\prime}\right) \sigma(m, z) \sigma\left(m^{\prime}, z\right) d m^{\prime} .
\end{array}
$$

Avant de nous occuper de la solution du problème (3.1)-(3.2), rappelons une propriété importante de l'opérateur intégral figurant au second membre de (3.1).

LEMME 3.1. Soit $\beta(\cdot, \cdot)$ la fonction introduite dans le paragraphe précédent. Alors, quelque soit $\sigma(\cdot) \in L^{1}\left(\mathbb{R}_{+}\right)$, on a

$$
\begin{aligned}
\int_{0}^{\infty} \frac{m}{2} \int_{0}^{m} \beta\left(m-m^{\prime}, m^{\prime}\right) \sigma\left(m^{\prime}\right) \sigma\left(m-m^{\prime}\right) d m^{\prime} d m+ \\
\\
-\int_{0}^{\infty} m \int_{0}^{\infty} \beta\left(m, m^{\prime}\right) \sigma(m) \sigma\left(m^{\prime}\right) d m^{\prime} d m=0 .
\end{aligned}
$$

DÉmonstration. En faisant le changement de variables

$$
q=m-m^{\prime}, \quad r=m^{\prime},
$$

dont le déterminant jacobien est égal à 1 , on a

$$
\int_{0}^{\infty} \frac{m}{2} \int_{0}^{m} \beta\left(m-m^{\prime}, m^{\prime}\right) \sigma\left(m^{\prime}\right) \sigma\left(m-m^{\prime}\right) d m^{\prime} d m=\int_{0}^{\infty} \int_{0}^{\infty} \frac{q+r}{2} \beta(q, r) \sigma(q) \sigma(r) d r d q .
$$

Par conséquent, compte tenu de la symétrie de la fonction $\beta$

$$
\beta(q, r)=\beta(r, q)
$$

on a

$$
\begin{aligned}
\int_{0}^{\infty} \int_{0}^{\infty} \frac{q+r}{2} \beta(q, r) \sigma(q) \sigma(r) d r d q= & \int_{0}^{\infty} \int_{0}^{\infty} q \beta(q, r) \sigma(q) \sigma(r) d r d q= \\
& =\int_{0}^{\infty} \int_{0}^{\infty} m \beta\left(m, m^{\prime}\right) \sigma(m) \sigma\left(m^{\prime}\right) d m^{\prime} d m,
\end{aligned}
$$

d'où on obtient (3.4). 
L'égalité (3.4) n'est autre que la loi de la conservation de la masse pour l'eau liquide contenue dans les gouttelettes.

Proposition 3.1. Soit $\bar{\sigma}(\cdot) \in L^{1}\left(\mathbb{R}_{+}\right)$avec $\operatorname{supp}(\bar{\sigma}) \subset\left[\bar{m}_{a}, \bar{m}_{A}\right]$. Alors le problème (3.1)-(3.2) admet une unique solution $\sigma \in C\left([0,1] ; L^{1}\left(\mathbb{R}_{+}\right)\right)$ (c'est-à-dire, l'application $z \longmapsto \sigma(\cdot, z)$ est une fonction continue de $[0,1] \grave{a}$ valeurs dans $L^{1}\left(\mathbb{R}_{+}\right)$).

DÉmonstration. Pour résoudre le problème (3.1)-(3.2), on considère $\sigma(\cdot, z)$ comme élément de $L^{1}\left(\mathbb{R}_{+}\right)$, de sorte que l'équation (3.3) peut être écrite dans la forme

$$
\frac{d \sigma}{d z}=F(\sigma)
$$

où

$$
\begin{array}{r}
F(\sigma)=F(\sigma)(m)=-\frac{m \alpha(m)}{2 g} \int_{0}^{m} \beta\left(m-m^{\prime}, m^{\prime}\right) \sigma\left(m^{\prime}\right) \sigma\left(m-m^{\prime}\right) d m^{\prime}+ \\
+\frac{m \alpha(m)}{g} \int_{0}^{\infty} \beta\left(m, m^{\prime}\right) \sigma(m) \sigma\left(m^{\prime}\right) d m^{\prime} .
\end{array}
$$

Posons

$$
C_{\beta}=\max \left[\sup _{0<m^{\prime}<m<\infty} \frac{m \alpha(m)}{2 g} \beta\left(m-m^{\prime}, m^{\prime}\right), \sup _{m, m^{\prime} \in \mathbb{R}_{+}} \frac{m \alpha(m)}{g} \beta\left(m, m^{\prime}\right)\right] .
$$

Alors, en rappelant l'expression de $F(\sigma)$, on a, pour $\sigma_{1}, \sigma_{2} \in L^{1}\left(\mathbb{R}_{+}\right)$,

$$
\begin{gathered}
\begin{array}{l}
\left\|F\left(\sigma_{1}\right)-F\left(\sigma_{2}\right)\right\|_{L^{1}\left(\mathbb{R}_{+}\right)}=\int_{0}^{\infty}\left|F\left(\sigma_{1}\right)(m)-F\left(\sigma_{2}\right)(m)\right| d m \leq \\
\leq C_{\beta} \int_{0}^{\infty} \int_{0}^{m} \mid \sigma_{1}\left(m^{\prime}\right)\left(\sigma_{1}\left(m-m^{\prime}\right)-\sigma_{2}\left(m-m^{\prime}\right)\right)+ \\
\quad+\left(\sigma_{1}\left(m^{\prime}\right)-\sigma_{2}\left(m^{\prime}\right)\right) \sigma_{2}\left(m-m^{\prime}\right) \mid d m^{\prime} d m+ \\
+C_{\beta} \int_{0}^{\infty} \int_{0}^{\infty} \mid\left(\sigma_{1}(m)\left(\sigma_{1}\left(m^{\prime}\right)-\sigma_{2}\left(m^{\prime}\right)\right)+\left(\sigma_{1}(m)-\sigma_{2}(m)\right) \sigma_{2}\left(m^{\prime}\right) \mid d m^{\prime} d m \leq\right. \\
\leq C_{\beta}\left(\left\|\sigma_{1} *\left(\left|\sigma_{1}-\sigma_{2}\right|\right)\right\|_{L^{1}}+\left\|\left(\left|\sigma_{1}-\sigma_{2}\right|\right) * \sigma_{2}\right\|_{L^{1}}\right)+ \\
+C_{\beta} \int_{0}^{\infty}\left(\left|\sigma_{1}(m)\right|\left\|\sigma_{1}-\sigma_{2}\right\|_{L^{1}}+\left|\sigma_{1}(m)-\sigma_{2}(m)\right|\left\|\sigma_{2}\right\|_{L^{1}}\right) d m \leq \\
\leq 2 C_{\beta}\left\|\sigma_{1}-\sigma_{2}\right\|_{L^{1}}\left(\left\|\sigma_{1}\right\|_{L^{1}}+\left\|\sigma_{2}\right\|_{L^{1}}\right)
\end{array}
\end{gathered}
$$


(pour la propriété de la convolution, voir par exemple [2]), ce qui montre que $F(\cdot)$ vérifie localement la condition de Lipchitz dans la topologie de $L^{1}\left(\mathbb{R}_{+}\right)$. Par conséquent, l'équation (3.5) avec la condition initiale (3.2) admet une solution $\sigma(\cdot, z)$ et une seule dans un intervalle $1-\delta \leq z \leq 1$ avec un $\delta>0$ suffisamment petit.

D'autre part, du lemme 3.1 et de l'équation (3.1) on déduit que

$$
\int_{0}^{\infty}\left(\sigma(m, z) \frac{g}{\alpha(m)}\right) d m=\int_{0}^{\infty}\left(\sigma(m, 1) \frac{g}{\alpha(m)}\right) d m,
$$

pourvu que $\sigma(\cdot, z)$ existe. Or, la condition (2.8) et l'hypothèse $\operatorname{supp}(\bar{\sigma}) \subset\left[\bar{m}_{a}, \bar{m}_{A}\right]$ impliquent que $\operatorname{supp}(\sigma(\cdot, z)) \subset\left[\bar{m}_{a}, \bar{m}_{A}\right]$. Donc, de la relation

$$
0<c_{1} \leq \frac{g}{\alpha(m)} \leq c_{2}<\infty \quad \forall m \in\left[\bar{m}_{a}, \bar{m}_{A}\right]
$$

avec deux constantes $c_{1}, c_{2}$ (qui résulte de l'hypothèse sur $\alpha(m)$ ), on déduit que $\|\sigma(\cdot, z)\|_{L^{1}\left(\mathbb{R}_{+}\right)}$est uniformément bornée en $z$ (pourvu que $\sigma(\cdot, z)$ existe), ce qui, joint à la condition de Lipschitz locale, nous donne la solution $\sigma(\cdot, z)$ de l'équation (3.5) dans tout l'intervalle [0,1]. La proposition est démontrée.

\section{Préliminaires pour le cas général}

Pour résoudre l'équation (2.4) avec la condition (2.5) $(\sigma(m, x, 1)=$ $\bar{\sigma}(m, x)$ ), nous allons utiliser l'idée de transformer l'équation (2.4) en une équation différentielle ordinaire, comme dans la démonstration de la proposition 3.1, où on a transformé l'équation (3.1) en (3.5). Pour cela, nous introduisons le changement de variables $(m, x, z) \mapsto(\tilde{m}, \xi, \tilde{z})$ défini par

$$
\left\{\begin{array}{l}
\tilde{m}=m \\
\xi=x-\bar{v} \frac{\alpha(m)}{g}(1-z), \\
\tilde{z}=z
\end{array}\right.
$$

et définissons

$$
\tilde{\sigma}(\tilde{m}, \xi, \tilde{z})=\sigma(m, x, z)=\sigma\left(m, \xi+\bar{v} \frac{\alpha(m)}{g}(1-z), z\right) .
$$

Dans la suite, toutefois, pour éviter la notation lourde, on va écrire simplement $m$ et $z$ au lieu de $\tilde{m}$ et $\tilde{z}$ et encore $\sigma(m, \xi, z)$ au lieu de 
$\tilde{\sigma}(\tilde{m}, \xi, \tilde{z})$, ce qui ne cause pas d'équivoque dans le calcul. Comme on peut le constater facilement, dans les coordonnées $(m, \xi, z)$ l'équation (2.4) se transforme en

$$
\frac{\partial}{\partial z} \sigma(m, \xi, z)=-\frac{m \alpha(m)}{2 g}
$$

$\cdot \int_{0}^{m} \beta\left(m-m^{\prime}, m^{\prime}\right) \sigma\left(m^{\prime}, \eta\left(m, m^{\prime}, \xi, z\right), z\right) \sigma\left(m-m^{\prime}, \eta\left(m, m-m^{\prime}, \xi, z\right), z\right) d m^{\prime}+$ $+\frac{m \alpha(m)}{g} \int_{0}^{\infty} \beta\left(m, m^{\prime}\right) \sigma(m, \xi, z) \sigma\left(m^{\prime}, \eta\left(m, m^{\prime}, \xi, z\right), z\right) d m^{\prime}$

où

$$
\eta\left(m, m^{\prime}, \xi, z\right)=\xi+\bar{v} \frac{\alpha(m)-\alpha\left(m^{\prime}\right)}{g}(1-z) .
$$

Pour réformuler l'équation $(4,1)$ en une équation différentielle ordinaire et établir des propriétés utiles de l'opérateur intégral du deuxième membre de cette équation, il nous convient, pour chaque $z \in[0,1]$ fixé, d'introduire la famille de courbes

$$
\gamma_{\tau}=\left\{(m, \xi) \in \mathbb{R}_{+} \times \mathbb{R} \mid \xi=\tau-\bar{v} \frac{\alpha(m)}{g}(1-z)\right\}, \quad \tau \in \mathbb{R}
$$

et de définir une mesure sur ces courbes.

Désignons par $P_{\mathbb{R}_{+}}$la projection de $\gamma_{\tau}$ sur $\mathbb{R}_{+}$, c'est-à-dire, pour les sous-ensembles $A^{\prime}$ de $\gamma_{\tau}$, on a

$$
P_{\mathbb{R}_{+}} A^{\prime}=\left\{m \in \mathbb{R}_{+} \mid \exists \xi \text { tel que }(m, \xi) \in A^{\prime}\right\} .
$$

La régularité de la fonction $\xi(m)=\tau-\bar{v} \frac{\alpha(m)}{g}(1-z)$ nous permet de définir les ensembles mesurables de $\gamma_{\tau}$ et la mesure $\mu_{\gamma}$ sur $\gamma_{\tau}$ par les relations suivantes :

i) $A^{\prime} \subset \gamma_{\tau}$ est mesurable si et seulement si $P_{\mathbb{R}_{+}} A^{\prime}$ est mesurable selon Lebesgue sur $\mathbb{R}_{+}$,

ii) $\mu_{\gamma}\left(A^{\prime}\right)=\mu_{L, \mathbb{R}_{+}}\left(P_{\mathbb{R}_{+}} A^{\prime}\right)$, où $\mu_{L, \mathbb{R}_{+}}(\cdot)$ est la mesure de Lebesgue sur $\mathbb{R}_{+}$.

Comme les courbes $\gamma_{\tau}, \tau \in \mathbb{R}$, sont parallèles (c'est-à-dire, définies par la translation de $\gamma_{0}$ par $\tau$ dans la direction de $\xi$, on voit immédiatement que la projection $P_{\mathbb{R}_{+}}$et la mesure $\mu_{\gamma}(\cdot)$ ne dépendent pas de $\tau \in \mathbb{R}$. 
La mesure $\mu_{\gamma}(\cdot)$ étant définie sur les courbes $\gamma_{\tau}$, nous allons éclaircir les relations entre $\mu_{\gamma}(\cdot)$ et la mesure sur $\mathbb{R}_{+} \times \mathbb{R}$. Pour ce faire, on pose

$$
\tau(m, \xi)=\xi+\bar{v} \frac{\alpha(m)}{g}(1-z)
$$

(c'est-à-dire, $\tau(m, \xi)$ est $\tau \in \mathbb{R}$ tel que $\left.(m, \xi) \in \gamma_{\tau}\right)$ et on considère la famille $\mathfrak{U}$ des ensembles $A$ ayant la forme

$$
A=\left\{(m, \xi) \in \mathbb{R}_{+} \times \mathbb{R} \mid m \in\left[m_{1}, m_{2}\left[, \tau(m, \xi) \in\left[\tau_{1}, \tau_{2}[,\}\right.\right.\right.\right.
$$

avec $0 \leq m_{1} \leq m_{2}<\infty,-\infty<\tau_{1} \leq \tau_{2}<\infty$. Si on définit la fonction $\tilde{\mu}: \mathfrak{U} \rightarrow \mathbb{R}_{+}$par la relation

$$
\tilde{\mu}(A)=\left(m_{2}-m_{1}\right)\left(\tau_{2}-\tau_{1}\right)
$$

pour $A=\left\{(m, \xi) \in \mathbb{R}_{+} \times \mathbb{R} \mid m \in\left[m_{1}, m_{2}\left[, \tau(m, \xi) \in\left[\tau_{1}, \tau_{2}[\}\right.\right.\right.\right.$, on constate que, de la même manière que la construction de la mesure de Lebesgue sur $\mathbb{R}_{+} \times \mathbb{R}$ à partir de la famille des rectangles, le prolongement de $\tilde{\mu}$ définit les ensembles mesurables selon $\tilde{\mu}$ de $\mathbb{R}_{+} \times \mathbb{R}$ et la mesure sur eux, mesure que nous notons toujours $\tilde{\mu}$, et que $\tilde{\mu}$ coïncide avec la mesure de Lebesgue $\mu_{L, \mathbb{R}_{+} \times \mathbb{R}} \operatorname{sur} \mathbb{R}_{+} \times \mathbb{R}$; on a en effet

$$
\tilde{\mu}(A)=\mu_{L, \mathbb{R}_{+} \times \mathbb{R}}(A)
$$

$\operatorname{pour} A \in \mathfrak{A}$.

Pour les mesures $\mu_{\gamma}$ et $\tilde{\mu}$ ainsi définies et les mesures de Lebesgue $\mu_{L, \mathbb{R}_{+}}, \mu_{L, \mathbb{R}}$ et $\mu_{L, \mathbb{R}_{+} \times \mathbb{R}}$ respectivement sur $\mathbb{R}_{+}, \mathbb{R}$ et $\mathbb{R}_{+} \times \mathbb{R}$, on a les relations suivantes.

Lemme 4.1. Soit A un ensemble mesurable (selon Lebesgue) de $\mathbb{R}_{+} \times \mathbb{R}$. On pose

$$
\begin{gathered}
A_{\tau}=\left\{m \in \mathbb{R}_{+} \mid \exists \xi \in \mathbb{R} \text { tel que }(m, \xi) \in \gamma_{\tau} \cap A\right\}, \\
A_{m}=\left\{\tau \in \mathbb{R} \mid \exists \xi \in \mathbb{R} \text { tel que }(m, \xi) \in \gamma_{\tau} \cap A\right\} .
\end{gathered}
$$

Alors on a

$$
\mu_{L, \mathbb{R}_{+} \times \mathbb{R}}(A)=\tilde{\mu}(A)=\int_{-\infty}^{\infty} \mu_{\gamma}\left(A_{\tau}\right) d \tau=\int_{\gamma_{0}} \mu_{L, \mathbb{R}}\left(A_{m}\right) \mu_{\gamma}(d m)=\int_{0}^{\infty} \mu_{L, \mathbb{R}}\left(A_{m}\right) d m
$$

(ici et dans la suite l'élément d'intégration par rapport à la mesure de Lebesgue s'écrit directement $d m, d \tau$ etc... sans utiliser les notations $\mu_{L, \mathbb{R}_{+}}(d m), \mu_{L, \mathbb{R}}(d \tau), \mu_{L, \mathbb{R}}(d \xi)$ etc...). 
LEMme 4.2. Soit $\sigma(m, \xi) \in L^{1}\left(\mathbb{R}_{+} \times \mathbb{R}\right)$. Alors, pour presque tout $\tau \in \mathbb{R}$ la restriction de $\sigma(m, \xi) \grave{a} \gamma_{\tau}$ appartient $\grave{a} L^{1}\left(\gamma_{\tau}, \mu_{\gamma}\right)$.

Lemme 4.3. Soit $\sigma(m, \xi) \in L^{1}\left(\mathbb{R}_{+} \times \mathbb{R}\right)$. Alors on $a$

$$
\begin{aligned}
& \int_{\mathbb{R}_{+} \times \mathbb{R}} \sigma(m, \xi) d m d \xi=\int_{\mathbb{R}_{+} \times \mathbb{R}} \sigma(m, \xi) d \tilde{\mu}= \\
& =\int_{-\infty}^{\infty}\left(\int_{\gamma_{\tau}} \sigma(m, \xi) \mu_{\gamma}(d m)\right) d \tau=\int_{\gamma_{0}}\left(\int_{-\infty}^{\infty} \sigma(m, \xi(m, \tau)) d \tau\right) \mu_{\gamma}(d m)= \\
& =\int_{0}^{\infty}\left(\int_{-\infty}^{\infty} \sigma(m, \xi) d \xi\right) d m=\int_{-\infty}^{\infty}\left(\int_{0}^{\infty} \sigma(m, \xi) d m\right) d \xi,
\end{aligned}
$$

$o \grave{u} \xi(m, \tau)=\tau-\bar{v} \frac{\alpha(m)}{g}(1-z)$.

Pour la démonstration des lemmes 4.1, 4.2 et 4.3, il suffit d'effectuer les modifications formelles nécessaires aux démonstrations des théorèmes classiques sur le produit des mesures et de Fubini (voir par exemple [8]), en tenant compte de la définition formulée ci-dessus des mesures $\mu_{\gamma}$ et $\tilde{\mu}$.

Maintenant on est en mesure de transformer l'équation (4.1) en une équation différentielle ordinaire. Pour cela on pose

$$
\tau(m, \xi, z)=\xi+\bar{v} \frac{\alpha(m)}{g}(1-z), \quad \gamma_{\tau}^{[0, m]}=\gamma_{\tau} \cap[0, m] \times \mathbb{R} .
$$

Cela étant, on peut écrire l'équation (4.1) dans la forme

$$
\frac{\partial}{\partial z} \sigma(z)=F_{z}(\sigma(z)), \quad \sigma(z)=\sigma(\cdot, \cdot, z),
$$

avec

$$
\begin{aligned}
& F_{z}(\sigma(z))= F_{z}(\sigma(z))(m, \xi)= \\
&=-\frac{m \alpha(m)}{2 g} \int_{\gamma_{\tau(m, \xi, z)}[0, m]} \beta\left(m-m^{\prime}, m^{\prime}\right) \sigma\left(m^{\prime}, \eta^{\prime}, z\right) \sigma\left(m-m^{\prime}, \eta^{\prime \prime}, z\right) \mu_{\gamma}\left(d m^{\prime}\right)+ \\
& \quad+\frac{m \alpha(m)}{g} \int_{\gamma_{\tau(m, \xi, z)}} \beta\left(m, m^{\prime}\right) \sigma\left(m^{\prime}, \eta^{\prime}, z\right) \sigma(m, \xi, z) \mu_{\gamma}\left(d m^{\prime}\right),
\end{aligned}
$$


où $\eta^{\prime}$ et $\eta^{\prime \prime}$ sont tels que

$$
\left(m^{\prime}, \eta^{\prime}\right) \in \gamma_{\tau(m, \xi, z)}, \quad\left(m-m^{\prime}, \eta^{\prime \prime}\right) \in \gamma_{\tau(m, \xi, z)} .
$$

L'équation (4.5) doit être envisagée avec la condition (2.5), c'est-à-dire

$$
\sigma(1)=\sigma(m, \xi, 1)=\bar{\sigma}(m, \xi) .
$$

\section{Existence et unicité de la solution dans le cas général}

Pour démontrer l'existence et l'unicité de la solution du problème (4.5)(4.6), nous avons besoin de préciser des conditions sur $\bar{\sigma}(m, \xi)$. Nous supposons que

$$
\begin{gathered}
\bar{\sigma}(\cdot, \cdot) \in L^{1}\left(\mathbb{R}_{+} \times \mathbb{R}\right) \cap L^{\infty}\left(\mathbb{R}_{+} \times \mathbb{R}\right), \\
\bar{\sigma}(m, \xi) \geq 0 \quad \text { p.p. dans } \mathbb{R}_{+} \times \mathbb{R} \\
\operatorname{supp}(\bar{\sigma}) \subset\left[\bar{m}_{a}, \bar{m}_{A}\right] \times \mathbb{R} \\
\|\bar{\sigma}\|_{L^{\infty}\left(\mathbb{R}_{+} \times \mathbb{R}\right)}<\frac{1}{M_{1}\left(\bar{m}_{A}-\bar{m}_{a}\right)}
\end{gathered}
$$

où

$$
M_{1}=\sup _{2 \bar{m}_{a} \leq m \leq \bar{m}_{A}, \bar{m}_{a} \leq m^{\prime} \leq m-\bar{m}_{a}} \frac{m \alpha(m)}{2 g} \beta\left(m-m^{\prime}, m^{\prime}\right) .
$$

Ici $\bar{m}_{a}$ et $\bar{m}_{A}\left(0<\bar{m}_{a}<\bar{m}_{A}<\infty\right)$ sont les deux nombres que l'on a introduits dans le paragraphe 2 . On a alors le résultat suivant.

Proposition 5.1. Si $\bar{\sigma}(m, \xi)$ satisfait aux conditions (5.1)-(5.4), alors l'équation (4.5) avec la condition (4.6) admet une solution $\sigma$ et une seule dans la classe

$$
\sigma \in C\left([0,1] ; L^{1}\left(\mathbb{R}_{+} \times \mathbb{R}\right)\right) \cap L^{\infty}\left(\mathbb{R}_{+} \times \mathbb{R} \times[0,1]\right) .
$$

Pour démontrer la proposition 5.1, commençons par la propriété de la convolution sur les courbes $\gamma_{\tau}$.

LEMme 5.1. Soient $f$ et $g$ deux fonctions appartenant $\grave{a} L^{1}\left(\gamma_{\tau}, \mu_{\gamma}\right)$. On pose

$$
(f * g)(m)=\int_{\gamma_{\tau}} f\left(m-m^{\prime}\right) g\left(m^{\prime}\right) \mu_{\gamma}\left(d m^{\prime}\right) .
$$


Alors on af*g $\in L^{1}\left(\gamma_{\tau}, \mu_{\gamma}\right)$ et

$$
\|f * g\|_{L^{1}\left(\gamma_{\tau}, \mu_{\gamma}\right)} \leq\|f\|_{L^{1}\left(\gamma_{\tau}, \mu_{\gamma}\right)}\|g\|_{L^{1}\left(\gamma_{\tau}, \mu_{\gamma}\right)} .
$$

Comme la mesure $\mu_{\gamma}$ ne dépend pas de $\tau$, le lemme 5.1 est vérifié de la même manière pour tous $\tau$.

DÉmonstration. La mesure $\mu_{\gamma}$ étant bien définie sur $\gamma_{\tau}$, le lemme se démontre de la même manière (avec des modifications purement formelles) que dans le cas des fonctions sommables par rapport à la mesure de Lebesgue (voir par exemple [2]).

A différence du cas $v=0$ (proposition 3.1) où on a considéré la solution $\sigma(\cdot, z)$ comme fonction de $z$ à valeurs dans $L^{1}\left(\mathbb{R}_{+}\right)$, pour la proposition 5.1 on a besoin de construire la solution $\sigma(\cdot, \cdot, z)$ comme fonction de $z$ à valeurs dans $L^{1}\left(\mathbb{R}_{+} \times \mathbb{R}\right) \cap L^{\infty}\left(\mathbb{R}_{+} \times \mathbb{R}\right)$. Pour ce faire, il nous convient d'examiner directement l'approximation successive avec laquelle on construit la solution $\sigma(m, \xi, z)$.

Posons

$$
\sigma^{[0]}(m, \xi, z)=\bar{\sigma}(m, \xi)
$$

et définissons $\sigma^{[n]}, n=1,2, \cdots$, par les relations

$$
\frac{\partial}{\partial z} \sigma^{[n]}=F_{z}\left(\sigma^{[n-1]}\right), \quad \sigma^{[n]}(m, \xi, 1)=\bar{\sigma}(m, \xi),
$$

où $F_{z}(\cdot)$ est l'opérateur défini dans (4.5).

LEMME 5.2. Quelque soit $n \in \mathbb{N}, \sigma^{[n]}$ est bien définie dans la classe

$$
\sigma^{[n]}(\cdot, \cdot, z) \in L^{1}\left(\mathbb{R}_{+} \times \mathbb{R}\right) \cap L^{\infty}\left(\mathbb{R}_{+} \times \mathbb{R}\right), \quad 0 \leq z \leq 1,
$$

et on a

$$
\operatorname{supp}\left(\sigma^{[n]}(\cdot, \cdot, z)\right) \subset\left[\bar{m}_{a}, \bar{m}_{A}\right] \times \mathbb{R} \quad \text { pour } 0 \leq z \leq 1,
$$

$\left\|\sigma^{[n]}(\cdot, \cdot, z)\right\|_{L^{\infty}\left(\mathbb{R}_{+} \times \mathbb{R}\right)} \leq \frac{\|\bar{\sigma}\|_{L^{\infty}\left(\mathbb{R}_{+} \times \mathbb{R}\right)}}{1-\left(M_{1}+M_{2}\right)\left(\bar{m}_{A}-\bar{m}_{a}\right)\|\bar{\sigma}\|_{L^{\infty}\left(\mathbb{R}_{+} \times \mathbb{R}\right)}(1-z)}$ $\operatorname{pour} \frac{\left(M_{1}+M_{2}\right)\left(\bar{m}_{A}-\bar{m}_{a}\right)\|\bar{\sigma}\|_{L^{\infty}\left(\mathbb{R}_{+} \times \mathbb{R}\right)}-1}{\left(M_{1}+M_{2}\right)\left(\bar{m}_{A}-\bar{m}_{a}\right)\|\bar{\sigma}\|_{L^{\infty}\left(\mathbb{R}_{+} \times \mathbb{R}\right)}}<z \leq 1$, où

$$
M_{2}=\sup _{m, m^{\prime} \in \mathbb{R}_{+}} \frac{m \alpha(m)}{g} \beta\left(m, m^{\prime}\right) .
$$


Démonstration. Remarquons d'abord que, si $\sigma^{[n]}(n \geq 1)$ est bien définie par les relations (5.8) et $\operatorname{si} \operatorname{supp}\left(\sigma^{[n-1]}(\cdot, \cdot, z)\right) \subset\left[\bar{m}_{a}, \bar{m}_{A}\right] \times \mathbb{R}$ pour $0 \leq z \leq 1$, alors $\sigma^{[n]}$ vérifie la condition (5.9). En effet, la condition (2.8) implique que la première intégrale de l'opérateur $F_{z}(\cdot)$ (voir (4.5)) s'annule pour $m \geq \bar{m}_{A}$. D'autre part, si $m<\bar{m}_{a}$, alors sous le signe d'intégration $\sigma^{[n-1]}\left(m-m^{\prime}, \cdot, z\right)$ et $\sigma^{[n-1]}\left(m^{\prime}, \cdot, z\right)$ s'annule et donc l'intégrale s'annule. En outre par hypothèse $\sigma^{[n-1]}$ s'annule pour $m<\bar{m}_{a}$ et $m>\bar{m}_{A}$, ce qui implique que même la seconde intégrale de l'opérateur $F_{z}(\cdot)$ s'annule pour $m<\bar{m}_{a}$ et $m>\bar{m}_{A}$. On en déduit (5.9) pour $\sigma^{[n]}$.

Examinons maintenant l'opérateur $F_{z}(\cdot)$ appliqué à $\sigma^{[n-1]}(\cdot, \cdot, z)$. En supposant que le support de $\sigma^{[n-1]}(\cdot, \cdot, z)$ est contenu dans $\left[\bar{m}_{a}, \bar{m}_{A}\right] \times \mathbb{R}$ et en rappelant (5.5) et (5.11), on a (avec la notation $\eta^{\prime}, \eta^{\prime \prime}$ comme dans (4.5))

$$
\begin{aligned}
& \left|\frac{m \alpha(m)}{2 g} \int_{\substack{\gamma_{\tau}[0, m] \\
\tau(m, \xi, z)}} \beta\left(m-m^{\prime}, m^{\prime}\right) \sigma^{[n-1]}\left(m^{\prime}, \eta^{\prime}, z\right) \sigma^{[n-1]}\left(m-m^{\prime}, \eta^{\prime \prime}, z\right) \mu_{\gamma}\left(d m^{\prime}\right)\right| \leq \\
& \leq M_{1}\left(\bar{m}_{A}-\bar{m}_{a}\right)\left\|\sigma^{[n-1]}(\cdot, \cdot, z)\right\|_{L^{\infty}\left(\gamma_{\tau(m, \xi, z)}, \mu_{\gamma}\right)}^{2}, \\
& \begin{array}{r}
\left|\frac{m \alpha(m)}{g} \int_{\gamma_{\tau(m, \xi, z)}} \beta\left(m, m^{\prime}\right) \sigma^{[n-1]}\left(m^{\prime}, \eta^{\prime}, z\right) \sigma^{[n-1]}(m, \xi, z) \mu_{\gamma}\left(d m^{\prime}\right)\right| \leq \\
\leq M_{2}\left(\bar{m}_{A}-\bar{m}_{a}\right)\left\|\sigma^{[n-1]}(\cdot, \cdot, z)\right\|_{L^{\infty}\left(\gamma_{\tau(m, \xi, z)}, \mu_{\gamma}\right)}^{2},
\end{array}
\end{aligned}
$$

où $M_{1}$ et $M_{2}$ sont les constantes définies dans (5.5) et (5.11) respectivement. On en déduit que, pour $\sigma^{[n]}$ définie par

$$
\sigma^{[n]}(m, \xi, z)=\bar{\sigma}(m, \xi)-\int_{z}^{1} F_{z^{\prime}}\left(\sigma^{[n-1]}\left(\cdot, \cdot, z^{\prime}\right)\right)(m, \xi) d z^{\prime},
$$

on a

$$
\begin{aligned}
& \left\|\sigma^{[n]}(\cdot, \cdot, z)\right\|_{L^{\infty}\left(\mathbb{R}_{+} \times \mathbb{R}\right)} \leq \\
\leq & \|\bar{\sigma}\|_{L^{\infty}\left(\mathbb{R}_{+} \times \mathbb{R}\right)}+\left(M_{1}+M_{2}\right)\left(\bar{m}_{A}-\bar{m}_{a}\right) \int_{z}^{1}\left\|\sigma^{[n-1]}\left(\cdot, \cdot, z^{\prime}\right)\right\|_{L^{\infty}\left(\mathbb{R}_{+} \times \mathbb{R}\right)}^{2} d z^{\prime} .
\end{aligned}
$$

En outre, en utilisant le lemme 5.1 et en tenant compte de la condition (2.8), on a 


$$
\begin{array}{r}
\left\|\frac{m \alpha(m)}{2 g} \int_{\gamma_{\tau}^{[0, m]}} \beta\left(m-m^{\prime}, m^{\prime}\right) \sigma^{[n-1]}\left(m^{\prime}, \eta^{\prime}, z\right) \sigma^{[n-1]}\left(m-m^{\prime}, \eta^{\prime \prime}, z\right) \mu_{\gamma}\left(d m^{\prime}\right)\right\|_{L^{1}\left(\gamma_{\tau}, \mu_{\gamma}\right)}+ \\
+\left\|\frac{m \alpha(m)}{g} \int_{\gamma_{\tau}} \beta\left(m, m^{\prime}\right) \sigma^{[n-1]}\left(m^{\prime}, \eta^{\prime}, z\right) \sigma^{[n-1]}(m, \eta, z) \mu_{\gamma}\left(d m^{\prime}\right)\right\|_{L^{1}\left(\gamma_{\tau}, \mu_{\gamma}\right)} \leq \\
\leq C\left\|\left.\sigma^{[n-1]}(\cdot, \cdot, z)\right|_{\gamma_{\tau}}\right\|_{L^{1}\left(\gamma_{\tau}, \mu_{\gamma}\right)}^{2},
\end{array}
$$

où $C$ est une constante indépendante de $z$ (et $\eta, \eta^{\prime}$ et $\eta^{\prime \prime}$ sont tels que $(m, \eta)$, $\left(m^{\prime}, \eta^{\prime}\right),\left(m-m^{\prime}, \eta^{\prime \prime}\right) \in \gamma_{\tau}$ comme dans (4.5)). Comme on a en outre

$$
\begin{aligned}
& \left\|\left.\sigma^{[n-1]}(\cdot, \cdot, z)\right|_{\gamma_{\tau}}\right\|_{L^{1}\left(\gamma_{\tau}, \mu_{\gamma}\right)} \leq\left(\bar{m}_{A}-\bar{m}_{a}\right)\left\|\left.\sigma^{[n-1]}(\cdot, \cdot, z)\right|_{\gamma_{\tau}}\right\|_{L^{\infty}\left(\gamma_{\tau}, \mu_{\gamma}\right)} \leq \\
& \left.\leq\left(\bar{m}_{A}-\bar{m}_{a}\right)\left\|\sigma^{[n-1]}(\cdot, \cdot, z)\right\|_{L^{\infty}\left(\mathbb{R}_{+} \times \mathbb{R}\right)} \quad \text { (pour presque tout } \tau \in \mathbb{R}\right),
\end{aligned}
$$

à l'aide du lemme 4.3 on en déduit que

$$
\begin{aligned}
& \left\|F_{z}\left(\sigma^{[n-1]}(\cdot, \cdot, z)\right)\right\|_{L^{1}\left(\mathbb{R}_{+} \times \mathbb{R}\right)} \leq \\
& \quad \leq C^{\prime}\left\|\sigma^{[n-1]}(\cdot, \cdot, z)\right\|_{L^{\infty}\left(\mathbb{R}_{+} \times \mathbb{R}\right)}\left\|\sigma^{[n-1]}(\cdot, \cdot, z)\right\|_{L^{1}\left(\mathbb{R}_{+} \times \mathbb{R}\right)}
\end{aligned}
$$

avec une constante $C^{\prime}$ indépendante de $z$.

Définissons une suite de fonctions $y_{n}(z), 0 \leq z \leq 1, n \in \mathbb{N}$, par les relations récursives

$$
\begin{gathered}
y_{0}(z)=\|\bar{\sigma}\|_{L^{\infty}\left(\mathbb{R}_{+} \times \mathbb{R}\right)} \quad \text { pour } 0 \leq z \leq 1 \\
y_{n}(z)=\|\bar{\sigma}\|_{L^{\infty}\left(\mathbb{R}_{+} \times \mathbb{R}\right)}+\left(M_{1}+M_{2}\right)\left(\bar{m}_{A}-\bar{m}_{a}\right) \int_{z}^{1} y_{n-1}\left(z^{\prime}\right)^{2} d z^{\prime} \\
\text { pour } 0 \leq z \leq 1, \quad n=1,2, \cdots .
\end{gathered}
$$

On va démontrer par l'induction mathématique que, quelque soit $n \in \mathbb{N}$, la fonction $\sigma^{[n]}$ est bien définie et vérifie, outre la condition (5.9), les relations

$$
\begin{gathered}
\left\|\sigma^{[n]}(\cdot, \cdot, z)\right\|_{L^{\infty}\left(\mathbb{R}_{+} \times \mathbb{R}\right)} \leq y_{n}(z), \\
\sup _{0 \leq z \leq 1}\left\|\sigma^{[n]}(\cdot, \cdot, z)\right\|_{L^{1}\left(\mathbb{R}_{+} \times \mathbb{R}\right)}<\infty .
\end{gathered}
$$

En effet, pour $n=0$, les relations (5.9), (5.16) et (5.17) résultent immédiatement de la définition (5.7) et des hypothèses (5.1) et (5.3). 
Supposons maintenant que $\sigma^{[n-1]}$ vérifie les relations (5.9), (5.16) et (5.17) (dans lesquelles on substitue naturellement $n-1$ à la place de $n$ ). Nous avons déjà remarqué que, dans ces hypothèse, $\sigma^{[n]}$ vérifie la condition (5.9). D'autre part, comme on le constate facilement, l'inégalité (5.16) résulte de la définition de $y_{n}$ et de l'inégalité (5.12). Enfin, l'inégalité (5.13), jointe à la définition (5.8) de $\sigma^{[n]}$ et l'hypothèse sur $\sigma^{[n-1]}$, implique que $\sigma^{[n]}$ vérifie également (5.17).

On remarque que la suite $\left\{y_{n}(z)\right\}_{n \in \mathbb{N}}, 0 \leq z \leq 1$, est une suite croissante et est l'approximation successive de la solution $Y(z)$ du problème de Cauchy (pour $z \leq 1$ )

$$
Y^{\prime}(z)=-\left(M_{1}+M_{2}\right)\left(\bar{m}_{A}-\bar{m}_{a}\right) Y(z)^{2}, \quad Y(1)=\|\bar{\sigma}\|_{L^{\infty}\left(\mathbb{R}_{+} \times \mathbb{R}\right)} .
$$

La fonction $Y(z)$ a la forme explicite

$$
Y(z)=\frac{\|\bar{\sigma}\|_{L^{\infty}\left(\mathbb{R}_{+} \times \mathbb{R}\right)}}{1-\left(M_{1}+M_{2}\right)\left(\bar{m}_{A}-\bar{m}_{a}\right)\|\bar{\sigma}\|_{L^{\infty}\left(\mathbb{R}_{+} \times \mathbb{R}\right)}(1-z)}
$$

$\operatorname{pour} \frac{\left(M_{1}+M_{2}\right)\left(\bar{m}_{A}-\bar{m}_{a}\right)\|\bar{\sigma}\|_{L^{\infty}\left(\mathbb{R}_{+} \times \mathbb{R}\right)}-1}{\left(M_{1}+M_{2}\right)\left(\bar{m}_{A}-\bar{m}_{a}\right)\|\bar{\sigma}\|_{L^{\infty}\left(\mathbb{R}_{+} \times \mathbb{R}\right)}}<z \leq 1$, ce qui nous permet de démontrer que (5.16) implique l'inégalité (5.10). Le lemme est démontré.

Maintenant nous allons démontrer la proposition 5.1.

DÉMONSTRATION DE LA PROPOSITION 5.1. Nous allons démontrer avant tout l'existence et l'unicité de la solution dans un intervalle $[1-\delta, 1]$ avec $\delta>0$ suffisament petit.

Considérons deux fonctions $\sigma_{1}$ et $\sigma_{2}$ appartenant à $L^{1}\left(\mathbb{R}_{+} \times \mathbb{R}\right)$ et la différence $F_{z}\left(\sigma_{1}\right)-F_{z}\left(\sigma_{2}\right)$. D'après le lemme 4.3 on a

$$
\begin{aligned}
\left\|F_{z}\left(\sigma_{1}\right)-F_{z}\left(\sigma_{2}\right)\right\|_{L^{1}\left(\mathbb{R}_{+} \times \mathbb{R}\right)} & =\int_{\mathbb{R}_{+} \times \mathbb{R}}\left|F_{z}\left(\sigma_{1}\right)-F_{z}\left(\sigma_{2}\right)\right| d m d \xi= \\
& =\int_{-\infty}^{\infty}\left(\int_{\gamma_{\tau}}\left|F_{z}\left(\sigma_{1}\right)-F_{z}\left(\sigma_{2}\right)\right| \mu_{\gamma}(d m)\right) d \tau .
\end{aligned}
$$

Or, on a

$$
\begin{aligned}
& \int_{\gamma_{\tau}}\left|F_{z}\left(\sigma_{1}\right)-F_{z}\left(\sigma_{2}\right)\right| \mu_{\gamma}(d m)= \\
& =\int_{\gamma_{\tau}}\left|\frac{m \alpha(m)}{2 g} \int_{\gamma_{\tau}^{[0, m]}} Q_{1}\left(m, m^{\prime}\right) \mu_{\gamma}\left(d m^{\prime}\right)-\frac{m \alpha(m)}{g} \int_{\gamma_{\tau}} Q_{2}\left(m, m^{\prime}\right) \mu_{\gamma}\left(d m^{\prime}\right)\right| \mu_{\gamma}(d m),
\end{aligned}
$$


où

$$
\begin{gathered}
Q_{1}\left(m, m^{\prime}\right)=\beta\left(m-m^{\prime}, m^{\prime}\right)\left(\sigma_{1}\left(m^{\prime}, \eta^{\prime}\right) \sigma_{1}\left(m-m^{\prime}, \eta^{\prime \prime}\right)-\sigma_{2}\left(m^{\prime}, \eta^{\prime}\right) \sigma_{2}\left(m-m^{\prime}, \eta^{\prime \prime}\right)\right), \\
Q_{2}\left(m, m^{\prime}\right)=\beta\left(m, m^{\prime}\right)\left(\sigma_{1}(m, \eta) \sigma_{1}\left(m^{\prime}, \eta^{\prime}\right)-\sigma_{2}(m, \eta) \sigma_{2}\left(m^{\prime}, \eta^{\prime}\right)\right), \\
(m, \eta),\left(m^{\prime}, \eta^{\prime}\right),\left(m-m^{\prime}, \eta^{\prime \prime}\right) \in \gamma_{\tau} \quad \text { (comme dans (4.5)). }
\end{gathered}
$$

Donc, en raisonnant de la même manière que dans (3.7) et en appliquant le lemme 5.1, on obtient

$$
\begin{aligned}
& \int_{\gamma_{\tau}}\left|F_{z}\left(\sigma_{1}\right)-F_{z}\left(\sigma_{2}\right)\right| \mu_{\gamma}(d m) \leq \\
& \leq 2 C_{\beta}\left\|\sigma_{1}-\sigma_{2}\right\|_{L^{1}\left(\gamma_{\tau}, \mu_{\gamma}\right)}\left(\left\|\sigma_{1}\right\|_{L^{1}\left(\gamma_{\tau}, \mu_{\gamma}\right)}+\left\|\sigma_{2}\right\|_{L^{1}\left(\gamma_{\tau}, \mu_{\gamma}\right)}\right),
\end{aligned}
$$

où $C_{\beta}$ est la constante définie dans (3.6). Encore une fois à l'aide du lemme 4.3, on déduit de (5.18) et (5.19) que

$$
\begin{aligned}
\| F_{z}\left(\sigma_{1}\right) & -F_{z}\left(\sigma_{2}\right) \|_{L^{1}\left(\mathbb{R}_{+} \times \mathbb{R}\right)} \leq \\
& \leq 2 C_{\beta} \operatorname{ess} \sup _{\tau \in \mathbb{R}}\left(\left\|\sigma_{1}\right\|_{L^{1}\left(\gamma_{\tau}, \mu_{\gamma}\right)}+\left\|\sigma_{2}\right\|_{L^{1}\left(\gamma_{\tau}, \mu_{\gamma}\right)}\right)\left\|\sigma_{1}-\sigma_{2}\right\|_{L^{1}\left(\mathbb{R}_{+} \times \mathbb{R}\right)} .
\end{aligned}
$$

Maintenant on substitue $\sigma_{1}=\sigma^{[n]}$ et $\sigma_{2}=\sigma^{[n-1]}$ dans (5.20). Alors en vertu de (5.10) (voir aussi (5.9)) on a

$$
\begin{aligned}
& \left\|F_{z}\left(\sigma^{[n]}\right)-F_{z}\left(\sigma^{[n-1]}\right)\right\|_{L^{1}(\mathbb{R}+\times \mathbb{R})} \leq \Lambda_{\sigma}(z)\left\|\sigma^{[n]}-\sigma^{[n-1]}\right\|_{L^{1}\left(\mathbb{R}_{+} \times \mathbb{R}\right)}, \\
& \Lambda_{\sigma}(z)=4 C_{\beta}\left(\bar{m}_{A}-\bar{m}_{a}\right) \frac{\|\bar{\sigma}\|_{L^{\infty}\left(\mathbb{R}_{+} \times \mathbb{R}\right)}}{1-\left(M_{1}+M_{2}\right)\left(\bar{m}_{A}-\bar{m}_{a}\right)\|\bar{\sigma}\|_{L^{\infty}\left(\mathbb{R}_{+} \times \mathbb{R}\right)}(1-z)} .
\end{aligned}
$$

C'est-à-dire, parmi les fonctions $\sigma^{[n]}, n \in \mathbb{N}$, l'opérateur $F_{z}(\cdot)$ satisfait à la condition de Lipschitz dans l'espace $L^{1}\left(\mathbb{R}_{+} \times \mathbb{R}\right)$ avec le coefficient de Lipschitz $\Lambda_{\sigma}(z)$. Donc, de la même manière que pour la démonstration de l'existence et de l'unicité de la solution locale d'une équation différentielle ordinaire, on peut démontrer qu'il existe un $\delta>0$ tel que $\sigma^{[n]}$ converge, quand $n$ tend vers l'infini, vers une fonction $\sigma$ dans la topologie de

$$
C\left([1-\delta, 1] ; L^{1}\left(\mathbb{R}_{+} \times \mathbb{R}\right)\right)
$$

et que la limite $\sigma$ satisfait, dans l'intervalle $[1-\delta, 1]$, à l'équation (4.5) et à la condition (4.6). On voit aisément que l'unicité de la solution $\sigma$ dans l'intervalle $[1-\delta, 1]$ se démontre d'une manière analogue à la démonstration du théorème classique.

Une fois obtenue la solution locale $\sigma$ dans l'intervalle [1 $-\delta, 1]$, examinons ses propriétés. Avant tout on remarque que (5.9) pour tout $n \in \mathbb{N}$ 
implique que la limite de la suite $\sigma^{[n]}$ jouit de la même propriété, c'est-àdire on a

$$
\operatorname{supp}(\sigma) \subset\left[\bar{m}_{a}, \bar{m}_{A}\right] \times \mathbb{R} \times[1-\delta, 1] .
$$

D'autre part, pourvu que $\sigma \geq 0$, la première intégrale de l'opérateur $F_{z}(\cdot)$ (voir (4.5)) est négative $(\leq 0)$, tandis que la seconde intégrale de $F_{z}(\cdot)$ est de la forme

$$
\sigma(m, \xi, z) \frac{m \alpha(m)}{g} \int_{\gamma_{\tau}} \beta\left(m, m^{\prime}\right) \sigma\left(m^{\prime}, \eta\left(m, m^{\prime}, \xi, z\right), z\right) \mu_{\gamma}\left(d m^{\prime}\right) .
$$

Donc de manière analogue aux cas des équations différentielles ordinaires, on peut démontrer que

$$
\sigma \geq 0 \quad \text { p.p. dans } \mathbb{R}_{+} \times \mathbb{R} \times[1-\delta, 1] .
$$

Les relations (5.22) et (5.23) étant démontrées, on peut refaire l'estimation de $\|\sigma(\cdot, \cdot, z)\|_{L^{\infty}\left(\mathbb{R}_{+} \times \mathbb{R}\right)}$. Pour cela on considère le deuxième membre $F_{z}(\sigma(z))$ de (4.5). En vertu de (5.23) on a

$$
\frac{m \alpha(m)}{g} \int_{\gamma_{\tau}(m, \xi, z)} \beta\left(m, m^{\prime}\right) \sigma\left(m^{\prime}, \eta^{\prime}, z\right) \sigma(m, \xi, z) \mu_{\gamma}\left(d m^{\prime}\right) \geq 0,
$$

ce qui nous permet de déduire de (4.5) que

$$
\frac{\partial}{\partial z} \sigma(m, \xi, z) \geq-\frac{m \alpha(m)}{2 g} \int_{\substack{[0, m] \\ \gamma \tau \tau, \xi, \xi,)}} \beta\left(m-m^{\prime}, m^{\prime}\right) \sigma\left(m^{\prime}, \eta^{\prime}, z\right) \sigma\left(m-m^{\prime}, \eta^{\prime \prime}, z\right) \mu_{\gamma}\left(d m^{\prime}\right)
$$

ou

$$
\frac{\partial}{\partial z} \sigma(m, \xi, z) \geq-M_{1} \int_{\substack{\left.\gamma_{\tau, 0}^{[0, m]}, \xi, z\right) \\ \gamma_{[(m, j)}}} \sigma\left(m^{\prime}, \eta^{\prime}, z\right) \sigma\left(m-m^{\prime}, \eta^{\prime \prime}, z\right) \mu_{\gamma}\left(d m^{\prime}\right)
$$

(ici $\eta^{\prime}$ et $\eta^{\prime \prime}$ sont comme dans (4.5)). Done, si on pose

$$
\varphi(z)=\|\sigma(\cdot, \cdot ; z)\|_{L^{\infty}\left(\mathbb{R}_{+} \times \mathbb{R}\right)},
$$

alors, compte tenu de (5.22), il résulte de (5.24) que

$$
\frac{\partial}{\partial z} \sigma(m, \xi, z) \geq-M_{1}\left(\bar{m}_{A}-\bar{m}_{a}\right) \varphi(z)^{2} \quad \text { p.p. dans } \mathbb{R}_{+} \times \mathbb{R},
$$


ou

$$
\sigma(m, \xi, z) \leq \bar{\sigma}(m, \xi)+M_{1}\left(\bar{m}_{A}-\bar{m}_{a}\right) \int_{z}^{1} \varphi\left(z^{\prime}\right)^{2} d z^{\prime} \quad \text { p.p. dans } \mathbb{R}_{+} \times \mathbb{R}
$$

d'où

$$
\varphi(z) \leq\|\bar{\sigma}\|_{L^{\infty}\left(\mathbb{R}_{+} \times \mathbb{R}\right)}+M_{1}\left(\bar{m}_{A}-\bar{m}_{a}\right) \int_{z}^{1} \varphi\left(z^{\prime}\right)^{2} d z^{\prime} .
$$

Cette inégalité implique que

$$
\|\sigma(\cdot, \cdot ; z)\|_{L^{\infty}\left(\mathbb{R}_{+} \times \mathbb{R}\right)}=\varphi(z) \leq \tilde{Y}(z)
$$

pour $z \leq 1$ dans l'intervalle de l'existence de $\sigma(\cdot, \cdot ; z)$ et de $\tilde{Y}(z)$, où $\tilde{Y}(z)$ est la solution de l'équation intégrale

$$
\tilde{Y}(z)=\|\bar{\sigma}\|_{L^{\infty}\left(\mathbb{R}_{+} \times \mathbb{R}\right)}+M_{1}\left(\bar{m}_{A}-\bar{m}_{a}\right) \int_{z}^{1} \tilde{Y}\left(z^{\prime}\right)^{2} d z^{\prime}
$$

ou, ce qui revient au même, du problème de Cauchy

$$
\frac{d \tilde{Y}(z)}{d z}=-M_{1}\left(\bar{m}_{A}-\bar{m}_{a}\right) \tilde{Y}(z)^{2}, \quad \tilde{Y}(1)=\|\bar{\sigma}\|_{L^{\infty}\left(\mathbb{R}_{+} \times \mathbb{R}\right)} .
$$

On a d'ailleurs

$$
\tilde{Y}(z)=\frac{\|\bar{\sigma}\|_{L^{\infty}\left(\mathbb{R}_{+} \times \mathbb{R}\right)}}{1-\|\bar{\sigma}\|_{L^{\infty}\left(\mathbb{R}_{+} \times \mathbb{R}\right)} M_{1}\left(\bar{m}_{A}-\bar{m}_{a}\right)(1-z)} .
$$

On rappelle que la condition (5.4) implique que le deuxième membre de (5.27) est bien défini pour tout $z \in[0,1]$. Donc l'inégalité (5.26) est valable pour tout $z \in[0,1]$ tel que $\sigma(\cdot, \cdot, z)$ existe.

Rappelons que l'on a construit la solution locale $\sigma(\cdot, \cdot ; z)$ dans un intervalle $[1-\delta, 1]$ et que l'on peut prolonger la solution $\sigma(\cdot, \cdot, z)$ pour tout l'intervalle où les conditions pour la construction de la solution locale continuent à être vérifiées. Or, de (5.20) on déduit que, si $\|\sigma(\cdot, \cdot ; z)\|_{L^{\infty}\left(\mathbb{R}_{+} \times \mathbb{R}\right)}<\infty$, alors on peut encore prolonger la solution. Par conséquent, en vertu de (5.26) et (5.27), la solution $\sigma(\cdot, \cdot, z)$ peut être prolongée dans tout l'intervalle $[0,1]$.

L'unicité de la solution résulte de l'unicité de la solution locale, ce qui achève la démonstration de la proposition.

Remerciement. Les auteurs tiennent à exprimer leur vive gratitude au Prof. M. Z. Aissaoui de l'Université 8 Mai 1945 de Guelma pour les discussions très fructueuses qu'ils ont eues avec lui tout le long de la réalisation du présent travail. 


\section{REFERENCES}

[1] H. Belhireche - M. Z. Aissaoui - H. Fujita Yashima, Equations monodimensionnelles du mouvement de l'air avec la transition de phase de l'eau. Sci. Techn. Univ. Constantine - A, 31 (2011), pp. 9-17.

[2] H. BREzIs, Analyse fonctionelle (Théorie et applications), Masson, Paris, 1983.

[3] M. Escobedo - S. Mischler - B. Perthame, Gelation in coagulation and fragmentation models. Comm. Math. Phys., 231 (2002), pp. 157-188.

[4] M. Escobedo - J. J. L. Velazquez, On the fundamental solution of a linearized homogeneous coagulation equation. Comm. Math. Phys., 297 (2010), pp. 759-816.

[5] M. Escobedo - S. Mischler - M. Rodriguez RicARd, On self-similarity and stationary problem for fragmentation and coagulation models. Ann. Inst. H. Poincaré, Anal. Non Linéaire, 22 (2005), pp. 99-125.

[6] H. Fujita Yashima - V. Campana - M. Z. Aissaoui, Système d'équations d'un modèle du mouvement de l'air impliquant la transition de phase de l'eau dans l'atmosphère. Ann. Math. Afr., 2 (2011), pp. 66-92.

[7] A. K. KıкoÏNE - I. K. KıкoÏNE, Physique moléculaire (traduit du russe). Mir, Moscou, 1979.

[8] A. N. Kolmogorov - S. V. Fomine, Eléments de la théorie des fonctions et de l'analyse fonctionelle (traduit du russe). Mir, Moscou, 1974.

[9] S. Mischler, Contributions à l'étude mathématique de quelques modèles issus de la physique hors équilibre. Thèse d'habilitation, Univ. Versailles Saint-Quentin, 2001.

[10] S. Mischler - M. Rodriguez RiCARD, Existence globale pour l'équation de Smoluchowski continue non homogène et comportement asymptotique des solutions. C. R. Acad. Sci. Paris, Sér. I, Math., 336 (2003), pp. 407-412.

[11] H. MÜLleR, Zur allgemeinen Theorie der raschen Koagulation. Kolloidchem. Beib., 27 (1928), pp. 223-250.

[12] B. Niethammer - J. J. L. Velazquez, Optimal bounds for self-similar solutions to coagulation equations with multiplicative kernel. A paraître sur Commun. PDE.

[13] F. Prodi - A. Battaglia, Meteorologia - Parte II, Microfisica. Grafica Pucci, Roma, 2004. (voir aussi le site: http://www.meteo.uni-bonn.de/mitarbeiter/ battaglia/teaching.html ).

[14] S. Selvaduray - H. Fujita Yashima, Equazioni del moto dell'aria con la transizione di fase dell'acqua nei tre stati: gassoso, liquido e solido. A paraître sur Memorie Accad. Sci. Torino.

[15] P.-X. Sheng - J.-T. MaO - J.-G. Li - A.-C. Zhang - J.-G. Sang - N.-X. PAn, Physique de l'atmosphère (en chinois). Publ. Univ. Pékin, Pékin, 2003.

[16] M. Smoluchowski, Drei Vorträge über Diffusion, Brownische Bewegung und Koagulation von Kolloidteilchen. Phys. Zeits., 17 (1916), pp. 557-585.

[17] V. M. Voloshtchuk, Théorie cinétique de coagulation (en russe). Gidrometeoizdat, Leningrad, 1984.

Manoscritto pervenuto in redazione il 22 Dicembre 2011. 